\title{
Athanase de Balad et la syllogistique catégorique d'Aristote en syriaque Analyse et étude comparative (Athanase, Probha et Paul le Perse, Pierre d'Espagne)
}

\author{
Jean-Pierre Levet \\ Université de Limoges
}

C'est animé par l'ambition d'apporter une contribution au développement d'une nouvelle philologie euro-méditerranéenne que l'on se propose d'étudier, à partir de passages d'une traduction française originale ${ }^{1}$, la partie du Compendium de logique qu'Athanase de $\mathrm{Balad}^{2}$, « modeste frère du saint monastère de Beit Malka », a consacré à la syllogistique catégorique d'Aristote.

En établissant une comparaison entre l'enseignement ainsi dispensé et les versions de Paul le Perse et de Probha, c'est à une civilisation grécoperso-syriaque que l'on souhaite s'intéresser pour en rappeler, après d'autres, l'existence, l'importance et le rayonnement dans l'ensemble du monde chamito-sémitique des premiers siècles de l'ère chrétienne, qui s'étendra ultérieurement à la sphère arabo-musulmane ${ }^{3}$, et pour montrer l'existence réelle et ancienne d'une communauté de pensée entre l'Orient et l'Occident médiévaux, en rapprochant les exemples choisis par les logiciens syriaques de ceux qui deviendront, en Occident, d'usage pédagogique courant à la suite des Summulae Logicales de Pierre d'Espagne'.

Autour du thème majeur de la recherche de la vérité, ce sont ainsi des langues appartenant à des familles différentes qui seront mises à contribution.

Précédée d'une brève présentation de la vie des quatre savants, l'analyse portera essentiellement sur les modes concluants et leur illustration, pour laquelle les traductions du texte grec ont dû être complétées par l'imagination des maîtres qui les ont élaborées, puisque Aristote se contente, sans proposer d'exemples concrets, de lettres symbolisant des termes.

Athanase de Balad, ville proche de l'actuelle Mossoul, fut patriarche 
jacobite de 684 à 687, année de sa mort. Formé au monastère de Qenneshre, fondé en 530 par Jean bar Aphtoma pour assurer, dans le monde syriaque, « la promotion des études grecques », il y suivit les leçons de Sévère Sebokt et eut pour condisciple Jacques d'Edesse ${ }^{5}$.

Là, il apprit à fonder ses travaux sur une grande rigueur philologique concernant le choix des mots et l'expression des concepts ${ }^{6}$. Elle n'interdit pas l'introduction volontaire d'innovations dans la matière traitée et dans les explications qui en sont données ou suggérées sous la forme d'un commentaire ou d'une paraphrase, dans un traité « simple et abrégé de la science syllogistique », alors que l'art de la logique «est difficile dans Aristote $»^{7}$.

De Paul le Perse, on retiendra qu'il dédia son traité de logique au roi sassanide Khosroès (531-579) et qu'il maîtrisait tout autant que le grec et le syriaque le pehlevi, si bien que son œuvre étend, en ce qui concerne l'Orient, les limites géographiques de l'aire concernée par cette étude à la Perse. Probha ${ }^{8}$, dont les dates de naissance et de mort sont incertaines (Ve ?/ VIe ?) fut prêtre et médecin en chef de l'hôpital d'Antioche.

Lui aussi homme de religion (il fut élu pape sous le nom de Jean XXI), Pierre d'Espagne, qui mourut en 1277, vécut dans la péninsule ibérique (Portugal et Espagne), en France et en Italie. Ses maîtres en logique furent un allemand et un anglais, saint Albert le Grand et William de Sherwood ${ }^{9}$. Ses Summulae Logicales, comme son livre de médecine, le Trésor des Pauvres, demeurèrent inscrits aux programmes des universités européennes jusqu'à la Renaissance et même au-delà ${ }^{10}$. L'œuvre de ce savant européen universel est donc parfaitement représentative de la logique médiévale de l'Occident.

Comme celles d'Athanase, de Probha et de Paul, sa syllogistique, et, de façon plus générale, sa logique reproduisent celles de l'Organon d'Aristote, avec la reprise d'une innovation très récente à son époque, que nous introduirons dans cet exposé, à savoir le codage par $\mathrm{A}, \mathrm{E}, \mathrm{I}$ et $\mathrm{O}$ des propositions en fonction de leur qualité et de leur quantité (A affirmative universelle, $\mathrm{E}$ négative universelle, I particulière affirmative, $\mathrm{O}$ particulière négative).

Cela facilitera la présentation des quatorze modes valides.

Mais examinons d'abord comment Athanase en introduit l'étude.

Avant de s'intéresser à la syllogistique aristotélicienne, Athanase propose, comme Aristote dans les deux premiers chapitres du livre I des Analytica Priora, des définitions de base concernant l'énoncé catégorique simple, l'affirmation et la négation, la quantification, les relations du carré 
logique (subalternation, contrariété, subcontrariété, contradiction), les termes, la démonstration, la conversion des prémisses, les énoncés modaux (Aristote I, 3) et les trois figures du syllogisme.

Sur le fond, le contenu est globalement celui du traité d'Aristote, mais le plan retenu, qui n'est pas celui du Stagirite, suit un ordre qui procède d'efforts pédagogiques, comme le montre l'illustration apportée par des exemples empruntés à Aristote ou imaginés par le traducteur lui-même.

Voici, en version française, avec les thèmes abordés, le texte d'Athanase, que l'on pourra aisément comparer avec l'original grec $^{11}$, avec des versions en latin (par exemple celles de Boèce) ${ }^{12}$, en syriaque ou dans une langue moderne.

\section{INTRODUCTION A LA SYLLOGISTIQUE}

\section{Définitions}

L'énoncé catégorique simple est soit une affirmation soit une négation. Celle-là est un discours prédicatif disant quelque chose de quelque chose ; celle-ci est un discours prédicatif qui nie quelque chose de quelque chose

Ces énoncés simples, l'affirmation et la négation, sont composés de deux termes, dont l'un est le sujet et l'autre le prédicat.

De ces deux termes, l'un est majeur ${ }^{13}$, l'autre mineur ${ }^{14}$. Celui qui a l'extension la plus grande est le prédicat, celui qui a l'extension la plus petite est le sujet.

Voici un exemple : l'homme marche . Les deux termes de cet énoncé sont homme et marche. Des deux termes qui le composent, le terme sujet est homme et le prédicat marche ${ }^{15}$.

Celui des termes qui, dans cet énoncé, exprime la substance, celui à propos duquel cet énoncé apporte une affirmation prédicative et auquel pourrait s'ajouter un quantificateur, celui dont il est dit quelque chose dans l'énoncé est le terme sujet. Un quantificateur serait tout, un (quelque) : tout homme ou un homme.

Le terme qui exprime le temps et auquel peut s'adjoindre le morphème de la négation, celui-là est le terme prédicat. Exemple : l'homme ne marche pas (l'homme est non marchant) ou l'homme n'est pas blanc (l'homme est non blanc). Dans les deux énoncés, le morphème négatif se place à côté du terme prédicat (et porte sur lui).

Parmi les propositions catégoriques, on distingue, pour ce qui est de la quantité, les universelles, les particulières et les indéterminées ${ }^{16}$.

9 
Les unes sont appelées majeures, les autres mineures ${ }^{17}$.

Est universelle celle qui possède un quantificateur universel, soit tout soit aucun (nul). Exemple : Tout homme est animal ${ }^{18}$; Nul homme n'est pierre $^{19}$.

Est particulière la proposition qui contient un quantificateur particulier, comme un, non tout, quelque. Exemple : Un homme est juste; Quelque homme est juste ${ }^{20}$.

Une proposition indéterminée est une proposition qui n'a aucun quantificateur, comme L'homme marche; L'homme ne marche pas. De ces deux propositions, aucune n'est déterminée. Elles ne contiennent ni tout, ni quelque, ni un, ni aucun.

Voilà pourquoi on ne se sert pas d'elles dans la syllogistique.

\section{Les relations entre propositions.}

Parmi les propositions affirmatives et négatives, quelques-unes ensemble disent le faux ${ }^{21}$; d'autres, au contraire, se répartissent entre 1'expression du vrai et celle du faux ${ }^{22}$. Sont ensemble fausses celles qui sont universelles. Exemple: Tout homme est blanc; Nul homme n'est blanc. Toutes les deux sont fausses. celle qui dit que Tout homme est blanc est fausse ; l'est également celle qui dit que Nul homme n'est blanc.

Sont vraies ensemble les particulières, celle qui énonce que Quelque homme est juste et celle qui dit que Quelque homme n'est pas juste. Toutes les deux disent ce qui est vrai et aucune d'entre elles n'est fausse.

Laissons de côté maintenant celles qui ensemble sont vraies et celles qui ensemble sont fausses. Intéressons-nous à celles qui s'opposent en ce qui concerne le vrai et le faux, l'une énonçant le vrai, l'autre le faux. Ce sont celles qui entrent dans un rapport de contradiction et sont situées dans des angles opposés <du carré logique> ${ }^{23}$. Exemple : Tout homme est blanc et Quelque homme n'est pas blanc. De ces deux propositions, l'une est vraie et l'autre fausse. Est vraie celle qui dit que Quelque homme n'est pas blanc; l'autre est fausse, celle qui dit que Tout homme est blanc. Autre exemple : celle qui dit que Aucun homme n'est juste et celle qui dit que Un homme est juste. Elles entrent également dans un rapport de contradiction et s'opposent entre elles : si l'une est vraie, l'autre est fausse.

C'est sur celles ${ }^{24}$ qui s'opposent ainsi que s'appuie toujours la démonstration apportée par les syllogismes. 


\section{Jean-Pierre Levet}

\section{La démonstration.}

Tout syllogisme est une démonstration, mais quelques démonstrations seulement sont des syllogismes.

Parmi les démonstrations, les unes sont paradigmatiques, d'autres épactiques, d'autres encore syllogistiques.

Toutes démontrent à partir de quelque chose de connu quelque chose d'inconnu. Si l'on cherchait à établir quelque chose qui n'est pas connu par soi-même par l'intermédiaire de quelque chose qui n'est pas connu, on aboutirait pareillement à l'inconnu; mais on réussit si l'on s'intéresse à quelque chose qui n'est ni connu ni évident par l'intermédiaire de quelque chose d'évident et de connu, qui nous est plus familier.

La démonstration paradigmatique est celle qui construit la démonstration sur la base d'exemples. Exemple : on aurait recours à une démonstration paradigmatique si l'on disait que l'on ne devrait pas donner une garde personnelle à Denys de Syracuse afin qu'il n'exerce pas la tyrannie une fois qu'il l'aurait reçue, et si, pour appuyer cela, on disait que Pisistrate a exercé la tyrannie après avoir été pourvu par les Athéniens d'une garde personnelle ${ }^{25}$.

Epactique se dit d'une démonstration qui procède par induction. Tout animal est doté de sens : l'homme, le cheval, le taureau, le chien sont sensibles; le sont tous les représentants d'une espèce animale. Par l'intermédiaire de l'induction et de la division en genres et en espèces, se démontre la proposition qui énonce que Tout animal est sensible. Une démonstration de cette nature est appelée inductive.

La démonstration syllogistique établit des énoncés particuliers à partir d'énoncés universels. Ce type de démonstration s'impose de manière plus nécessaire et plus convaincante que les autres. Il procède de la nécessité. Alors qu'aux autres s'attache la persuasion, à lui s'attache la vérité ${ }^{26}$. Exemple : si l'on dit que La justice est un bien, on le démontre en passant par le beau : La justice est belle, or Toute chose belle est un bien, donc La justice est un bien ${ }^{27}$. Si l'on dit que Aucun homme n'est pierre, on le démontre à partir de la sensibilité de l'animal : Tout homme est sensible, Tout homme est animal, Aucune pierre n'est animal. Semblablement la pierre n'est pas sensible, aucun homme n'est donc une pierre ${ }^{28}$. Les deux raisonnements sont syllogistiques. L'un aboutit à une conclusion affirmative, l'autre à une conclusion négative ${ }^{29}$.

La justice est un bien est établi par le beau et tout beau est de manière 11 
= Athanase de Balad et la syllogistique catégorique d'Aristote en syriaque

affirmative un bien. Aucun homme n'est une pierre, cette conclusion négative est construite sur un raisonnement impliquant la sensibilité de l'animal. L'un a quelque chose, l'autre ne l'a pas. L'homme a de la sensibilité parce qu'il est animal, mais la pierre n'en a pas.

Puisque le syllogisme est la démonstration de quelque chose d'inconnu par l'intermédiaire de quelque chose qui est admis, notre exposé traitera des termes contenus dans les prémisses et des prémisses ellesmêmes.

\section{Les termes et les prémisses, généralités sur la conversion des prémisses.}

Toute prémisse catégorique ou problématique est composée de deux termes, le sujet et le prédicat, dont l'un est le majeur et l'autre le mineur. Est majeur le prédicat, le sujet étant mineur. C'est là ce qui caractérise chacun d'entre eux pour ce qui est de son rôle dans la prémisse, mais, en tant que termes indépendants, aucun n'est plus que l'autre.

Il en va de même pour les prémisses. Une est la majeure, l'autre la mineure. Est majeure celle qui contient le terme majeur, est mineur celle qui contient le mineur ${ }^{30}$.

Parmi les prémisses, les unes peuvent se convertir, les autres ne le peuvent pas. La conversion d'une prémisse se fait par l'inversion des termes. Il y a interversion des termes lorsque le terme prédicat devient sujet et que le terme sujet devient prédicat. Les propositions sont de même qualité et vraies conjointement ${ }^{31}$.

Sont dites ayant la même qualité les propositions négatives ensemble et de la même manière les propositions affirmatives conjointement.

Voici comment on procède à une conversion : Aucun cheval n'est homme devient Aucun homme n'est cheval. Toutes les deux sont vraies et négatives, les mêmes termes figurent dans les deux, mais pas de la même façon, puisqu'il y a interversion. Dans l'une, homme est sujet et le prédicat est cheval ; dans l'autre, homme est prédicat, alors que le sujet est cheval. ce ne sont donc pas les mêmes propositions.

\section{Les propositions modales.}

Les propositions sont seulement catégoriques ou modales, la modalité étant, par exemple, le nécessaire ou le possible ${ }^{32}$. Les propositions nécessaires sont celles dans lesquelles à être, <verbe copule> s'ajoute toujours. Exemple : Tout homme est animal : cela relève du nécessaire et de 
ce qui est toujours. Le possible est ce qui peut arriver, comme L'homme, quand il a soixante ans, est blanc ${ }^{33}$.

Les propositions nécessaires se convertissent, les possibles ne le font pas.

\section{Les règles de conversion.}

L'universelle affirmative ne se convertit pas <parfaitement>. Il en va de même pour celle qui est nécessaire comme pour celle qui est possible.

Si la proposition énonçant que Tout homme est animal est vraie, est fausse celle qui énonce que Tout animal est nécessairement homme. Cette règle s'applique toutes les fois que la substance est en cause. la négation de la proposition convertie serait vraie, Tout animal n'est pas homme.

Lorsqu'il s'agit, en revanche, du propre, la proposition résultant de la conversion est également vraie. Exemple : Tout homme est animal capable de rire. Est également vraie la proposition Tout animal capable de rire est homme. La conversion de l'affirmative universelle ne va pas de soi. Les termes doivent être les mêmes pour ce qui est de toute la substance.

L'affirmative particulière se convertit parfaitement et l'affirmative universelle se convertit imparfaitement en particulière affirmative. Est vraie la proposition selon laquelle Un (quelque) homme est animal. Est tout aussi vraie celle selon laquelle Un (quelque) animal est homme.

Si une affirmative universelle est vraie, la particulière affirmative qui lui correspondra après conversion sera vraie également, qu'elle soit catégorique, nécessaire ou possible. Si est vraie Tout homme est animal, est également vraie la proposition qui énonce que Un (quelque) animal est homme.

Les négatives universelles se convertissent parfaitement, qu'elles soient purement catégoriques ou qu'elles relèvent de la modalité du possible.

La particulière négative, en revanche, ne se convertit pas, ni en ellemême ni sous une quelconque autre forme. Si Quelque animal n'est pas homme est vraie, n'est pas vraie la proposition $^{34}$ qui énoncerait que L'homme n'est pas animal, mais pourtant Quelque homme est animal.

\section{Les figures}

Parlons maintenant des figures et de leur composition. Elles sont au nombre de trois ${ }^{35}$. Chacune d'entre elles se compose de deux prémisses et 1 T 
de trois termes. Le moyen terme est celui qui apparaît deux fois, il est associé aux deux extrêmes. Avec eux, il forme les deux prémisses, qui constituent le syllogisme simple.

Parmi les syllogismes, les uns sont simples, les autres sont composés. Sont simples ceux qui sont faits de deux prémisses, les autres sont composés ${ }^{36}$.

Le moyen terme est celui par lequel passe la démonstration de ce qui est ignoré dans le problème posé. Il peut se placer de trois façons seulement. Ou il est sujet par rapport aux deux extrêmes ou il est prédicat des deux extrêmes ou encore sujet par rapport à l'un et prédicat par rapport à l'autre ${ }^{37}$.

On appelle extrêmes les deux termes qui sont associés à ce moyen. Quand le moyen s'associe à ces extrêmes, cela forme les deux prémisses.

Lorsque le moyen est prédicat par rapport aux deux extrêmes, se constitue la deuxième figure. La deuxième figure est celle dans laquelle le moyen terme est prédicat dans les deux prémisses.

La troisième figure apparaît quand le moyen terme est sujet par rapport aux deux extrêmes.

S'il est sujet par rapport à l'un des extrêmes et prédicat de l'autre, se construit la première figure, qui ne nécessite pas de démonstration ${ }^{38}$. La première figure est faite de deux prémisses dans lesquelles le moyen, qui est moyen, par position occupe vraiment la place qui doit être la sienne, puisque, dans l'une des prémisses, il est prédicat et, dans l'autre, sujet. Exemple : Tout homme est animal, Tout animal est substance ${ }^{39}$. Animal apparaît dans les deux prémisses, il est moyen par position. A partir de cela, on conclut ceci : Tout homme est substance. Puisque Tout animal est substance, c'est animal qui est sous substance. Sous animal est homme puisque Tout homme est animal ${ }^{40}$. Telle est la conclusion et telle est la position respective des trois termes dans la première figure.

Elle a quatre modes valides, dont les conclusions sont une négative universelle, une affirmative universelle, une particulière affirmative, une particulière négative. Tels sont les syllogismes parfaits, concluants dans la première figure, qui n'ont pas besoin de démonstration. On dit qu'ils sont parfaits parce qu'ils n'ont pas besoin d'une démonstration complémentaire. Ils sont clairs par eux-mêmes et leur démonstration est évidente.

\section{Eléments additionnels : choix des termes, polysémie etc.}

Athanase, après avoir exposé les modes valides du syllogisme d'une manière que l'on va présenter dans la seconde partie de cette étude, ajoute quelques informations générales supplémentaires. Elles correspondent à 


\section{Jean-Pierre Levet}

l'enseignement d'Aristote, brièvement résumé, puisé tant dans les Analytiques que dans les Réfutations Sophistiques. En voici une partie significative.

Pour choisir les termes, il faut examiner pour chacun d'eux ce qui s'attache à lui et ce qu'il n'est pas. A animal s'attachent la substance et la sensibilité : Tout animal est substance; Tout animal est sensible; Aucun animal n'est inanimé; Aucun animal n'est pierre; Aucune pierre n'est animal. Pour tous les termes, il convient de procéder de cette façon, afin de savoir ce qui est vrai. Il faut donc choisir les termes comme nous l'avons fait pour ceux que nous avons posés et construire de cette manière les prémisses et le syllogisme.

C'est ainsi que nous construirons des syllogismes véridiques. Lorsque les prémisses seront vraies, les syllogismes aussi le seront ${ }^{41}$.

Si un mot a plusieurs sens, ce n'est pas une prémisse que l'on peut construire, mais plusieurs.

A partir de toute affirmation, on est en mesure de bâtir une négation. Exemple : Socrate est blanc. La négation de cet énoncé est Socrate n'est pas blanc. On est fondé à procéder dans tous les cas de la même façon.

Ce qui devient, devient de cinq manières, par changement de forme, par composition, par diminution, par accroissement ou par transformation. la pensée.

Les arguments sophistiques s'appuient soit sur l'information soit sur

On ne doit pas recourir à une proposition indéterminée ${ }^{42}$, mais à une universelle ou à une particulière.

Ces informations sont suivies d'un développement sur la raisonnement par l'impossible. Son enseignement sera replacé à part, comme Athanase l'a voulu, après la présentation du second mode valide qu'il concerne.

\section{Bilan}

Si l'on dresse un bilan de l'ensemble de ces considérations générales, on ne peut que constater que la rédaction d'Athanase s'apparente plus, tant par le plan choisi que par le contenu exposé, à une réécriture complète, à partir de matériaux puisés essentiellement dans les premiers chapitres des Analytica Priora d'Aristote qu'à une traduction. Le maître syriaque écrit un manuel original, synthétique, en puisant dans la matière aristotélicienne.

La partie principale de son livre porte évidemment sur les modes valides. Athanase les étudie directement, après avoir opéré un tri entre 10 
modes valides et modes invalides, qui ne sont pas évoqués. Il se différencie ainsi nettement d'Aristote et de Paul le Perse, qui procède, lui, à une présentation, traduite d'Aristote, de tous les modes théoriquement concevables, avant de dire, à propos de chacun d'entre eux, s'il est concluant.

La méthode d'Athanase est aussi celle de Probha. Ce dernier et Paul le Perse bâtissent deux sortes de syllogismes, les uns codés avec des lettres, comme Aristote l'avait fait, alors que le Stagirite s'était contenté d'indiquer, pour les modes invalides, des termes impliquant une attribution et d'autres une absence d'attribution.

Paul et Probha écrivent leurs syllogismes codés dans l'ordre majeure/mineure, que Paul suit également de manière systématique pour les exemples pratiques. Probha est plus hésitant, puisqu' il préfère l'ordre inverse mineure/majeure le plus souvent, sans que toutefois ce choix soit exclusif $^{43}$. Dans l'exposé comparatif, on rétablira, pour faciliter la lecture, l'ordre majeure/mineure dans tous les syllogismes de Probha et l'on respectera ce même ordre, qui caractérise les syllogismes de Paul le Perse et de Pierre d'Espagne.

Vraisemblablement parce qu'il a enseigné dans son introduction que tout syllogisme est application d'un cas particulier à un énoncé général, Athanase respecte l'ordre mineure/majeure, suivant sur ce point une tradition postérieure à Aristote, qui se manifeste notamment dans de nombreux syllogismes de Galien.

Pour la commodité de l'analyse, on respectera la disposition d'Athanase dans la version française, mais on ajoutera une présentation majeure/mineure pour faciliter la compréhension des données comparatives.

Tout ce que l'on vient de dire à propos de Probha, de Paul et d'Athanase confirme bien que leurs traductions des Analytiques d'Aristote sont plus libres que strictes. Ce ne sont pas vraiment des traductions stricto sensu, mais plutôt des reprises du texte traduit et remodelé pour servir de base à un enseignement dispensé par ces maîtres. Traduite en syriaque, l'œuvre d'Aristote n'est pas figée, elle vit d'une existence nouvelle, qui respecte son contenu scientifique, mais adapte sa présentation à des pratiques pédagogiques qui portent la marque propre de chaque auteur.

Ces textes, comme celui de Pierre d'Espagne, nous introduisent donc dans le courant dynamique de la tradition logique, à la fois respectueux du texte fondateur et novateur dans la mesure où il peut l'être, c'est-à-dire dans l'illustration concrète des règles intangibles. C'est là du moins ce que l'on souhaiterait montrer. 


\section{Jean-Pierre Levet}

\section{Les modes valides : traduction du texte d'Athanase et commentaire comparatif.}

Revenons donc au texte d'Athanase

\section{Première figure}

\section{Premier mode de la première figure}

\section{Texte d'Athanase:}

Le premier mode est celui qui, à partir d'une majeure affirmative universelle et d'une affirmative universelle comme mineure a pour conclusion une affirmative universelle, puisque les termes sont dans les rapports qui ont été indiqués ${ }^{44}$ :

Tout homme est animal

Tout animal est sensible

Tout homme est sensible

soit

Tout animal est sensible

Tout homme est animal

Tout homme est sensible

Athanase a déjà cité un autre syllogisme du même mode :

Tout animal est substance

Tout homme est animal

Tout homme est substance

texte de Paul :

Tout animal est substance

Tout homme est animal

Tout homme est substance

texte de Probha :

Tout animal est substance

Tout homme est animal

Tout homme est substance

Texte de Pierre d'Espagne :

Tout animal est substance

Tout homme est animal

Tout homme est substance

Le même exemple est proposé par les quatre logiciens. On est IV 
d'autant plus fondé à le considérer comme canonique que Galien ${ }^{45}$ 1'utilise également.

Athanase ajoute ceci : ce mode conclut toujours par une affirmative universelle à partir de deux affirmatives universelles. Il est le premier mode parfait.

\section{Deuxième mode de la première figure}

Texte d'Athanase :

Le deuxième mode est celui qui, à partir d'une universelle affirmative comme mineure et d'une négative universelle comme majeure a pour conclusion une négative universelle ${ }^{46}$ :

Tout homme est animal

Aucun animal n'est pierre

Aucun homme n'est pierre

soit

Aucun animal n'est pierre

Tout homme est animal

Aucun homme n'est pierre

Texte de Paul :

Aucun arbre n'est cheval

Tout olivier est arbre

Nul olivier n'est cheval

Texte de Probha :

Aucun animal n'est pierre

Tout homme est animal

Aucun homme n'est pierre

Texte de Pierre :

Aucun animal n'est pierre

Tout homme est animal

Aucun homme n'est pierre

Trois des quatre logiciens recourent au même exemple. Seul Paul propose un syllogisme différent.

\section{Troisième mode de la première figure}

Le troisième mode est celui qui, à partir d'une affirmative universelle comme majeure et d'une particulière affirmative comme mineure, a pour conclusion une particulière affirmative ${ }^{47}$.

Texte d'Athanase :

Quelque être vivant est animal

Tout animal est sensible

Quelque animal est sensible 


\section{Jean-Pierre Levet}

soit

Tout animal est sensible

Quelque être vivant est animal

Quelque animal est sensible

Texte de Paul :

Tout homme est capable de rire

Quelque animal est homme

Quelque animal est capable de rire

Texte de Probha :

Tout homme est mortel

Quelque être doté de raison est homme

Quelque être doté de raison est mortel

Texte de Pierre :

Tout animal est substance

Quelque homme est animal

Quelque homme est substance

Chaque logicien a son propre exemple. On constate cependant la présence de termes communs à trois auteurs chacun : animal et homme.

Athanase ajoute la remarque suivante : ce syllogisme est également parfait. Il est clair que ce qu'a tout animal, l'être vivant l'a aussi puisqu'il est sous animal ${ }^{48}$.

\section{Quatrième mode de la première figure}

Le quatrième mode est celui dans lequel, à partir d'une majeure négative universelle et d'une mineure particulière affirmative, on obtient comme conclusion une particulière négative ${ }^{49}$. Construisons les prémisses de la façon indiquée pour donner un exemple :

Texte d'Athanase :

Quelque être vivant est animal

Aucun animal n'est pierre

Quelque être vivant n'est pas pierre

soit

Aucun animal n'est pierre

Quelque être vivant est animal

Quelque être vivant n'est pas pierre

Texte de Paul :

Nul cheval n'est capable de rire

Quelque animal est cheval

Quelque animal n'est pas capable de rire

19 
Texte de Probha:

Nul philosophe n'est inculte

Quelque homme est philosophe

Quelque homme n'est pas inculte

Texte de Pierre :

Nul animal n'est pierre

Quelque homme est animal

Quelque homme n'est pas pierre

Les syllogismes de Paul et de Probha sont originaux, alors que ceux d'Athanase et de Pierre se ressemblent au point qu'il n'est pas impossible qu'ils remontent à un ancêtre commun, identique à celui de Pierre et intelligemment modifié par Athanase par substitution dans la mineure d'un énoncé nouveau à la subalterne d'une universelle de nature à surprendre un élève débutant.

Athanase complète son texte par un constat. Tels sont les quatre modes parfaits de la première figure. Ce qui caractérise cette première figure, c'est d'avoir une mineure toujours affirmative et un moyen terme toujours moyen par sa position.

\section{Deuxième figure}

La deuxième figure se définit par la présence d'un moyen terme toujours supérieur <en extension> aux deux extrêmes et prédicat de chacun des deux. Cette figure aussi a quatre modes concluants, dont deux ont pour conclusions des universelles, les autres ayant pour conclusions des particulières.

Ce qui caractérise cette figure, c'est que les prémisses sont différentes par la qualité, puisque l'une est négative et que l'autre, en revanche, est affirmative, et que, d'autre part, la majeure est toujours universelle.

En associant les termes de cette manière et en conservant leur caractère propre <leur extension>, on forme les modes de la deuxième figure.

\section{Premier mode de la deuxième figure}

Le premier mode de la deuxième figure est celui qui se construit à partir d'une majeure négative universelle et d'une affirmative universelle comme mineure. la conclusion obtenue est une universelle négative ${ }^{50}$. figure $^{51}$

Si l'on convertit la majeure, on retrouve un mode de la première

Exemple

texte d'Athanase : 
Tout homme est animal

Nulle pierre n'est animal

Nul homme n'est pierre

soit

Nulle pierre n'est animal

Tout homme est animal

Nul homme n'est pierre

Texte de Paul :

Nul cheval n'est doué de raison

Tout homme est doué de raison

Nul homme n'est cheval

texte de Probha :

Nulle pierre n'est animal

Tout homme est animal

Nul homme n'est pierre

texte de Pierre :

Nulle pierre n'est animal

Tout homme est animal

Nul homme n'est pierre

Paul est le seul à recourir à un syllogisme entièrement recomposé. les trois autres auteurs adaptent, avec le même texte, leur syllogisme à une réduction facile à celui qu'ils ont proposé pour illustrer le deuxième mode de la première figure. La concordance est donc la même que celle qui a été constatée.

Athanase conclut par la réduction à la première figure. Par conversion de la majeure, à partir de Aucune pierre n'est animal, on obtient Aucun animal n'est pierre.

\section{Deuxième mode de la deuxième figure}

Le deuxième mode de la deuxième figure est celui qui, à partir d'une mineure négative universelle et d'une majeure affirmative universelle, a pour conclusion une négative universelle ${ }^{52}$. Par conversion de la mineure et de la conclusion, on retrouve la première figure ${ }^{53}$.

texte d'Athanase :

Nul cheval n'est capable de rire

Tout homme est capable de rire

Nul cheval n'est homme

soit

Tout homme est capable de rire 
Nul cheval n'est capable de rire

Nul cheval n'est homme

texte de Paul :

Tout être instruit est homme

Nul cheval n'est homme

Nul cheval n'est instruit

texte de Probha :

Tout homme est animal

Nulle pierre n'est animal

Nulle pierre n'est homme

texte de Pierre :

Tout homme est animal

Nulle pierre n'est animal

Nulle pierre n'est homme

Probha et Pierre ont choisi le même exemple, qui leur permet de suggérer directement une réduction au deuxième mode de la première figure. Athanase a préféré innover pour ne pas retenir successivement deux raisonnements semblables. Paul également a fait preuve d'imagination. On remarquera la présence de termes identiques dans les syllogismes de Paul et d'Athanase (cheval et homme). Faut-il l'interpréter comme une coïncidence ou faut-il supposer l'existence d'une source commune figurant dans un corpus classique? La seconde branche de l'alternative paraît plus vraisemblable.

Athanase, avant de passer au troisième mode, revient sur la réduction, dont il explicite le mécanisme, moins évident que s'il avait retenu le même syllogisme que Probha. Les lignes qui suivent sont donc l'indice sûr d'une prise de liberté par rapport à une simple traduction et d'un effort de réécriture et conjointement d'innovation.

En convertissant la négative universelle, celle qui dit que Aucun cheval n'est <un être > capable de rire, en énonçant donc que Aucun être capable de rire n'est cheval, on retrouve le deuxième mode de la première figure, Tout homme est capable de rire; Aucun être capable de rire n'est cheval, et de là on conclut que Aucun homme n'est cheval. On retrouve le bon ordre des termes en convertissant la négative universelle <qui constitue la conclusion>. Le moyen terme occupe les positions qui sont les siennes dans le deuxième mode de la première figure.

Troisième mode de la deuxième figure

Le troisième mode de la deuxième figure est celui qui, à partir d'une majeure négative universelle et d'une mineure particulière affirmative, 
aboutit à une conclusion particulière négative. En convertissant la majeure, on retrouve la structure de la première figure ${ }^{54}$.

texte d'Athanase

Quelque être animé est animal

Aucune pierre n'est animal

Quelque être animé n'est pas pierre

soit

Aucune pierre n'est animal

Quelque être vivant est animal

Quelque être vivant n'est pas pierre

texte de Paul

Nul oiseau n'est cheval

Quelque animal est cheval

Quelque animal n'est pas cheval

texte de Probha

Nul homme n'est cheval

Quelque être mortel est cheval

Quelque être mortel n'est pas homme

texte de Pierre

Nulle pierre n'est animal

Quelque homme est animal

Quelque homme n'est pas pierre

Une fois de plus, les exemples d'Athanase et de Pierre sont proches et permettent un renvoi direct au quatrième mode de la première figure. Probha et Paul ont innové, sans se soucier de manifester directement le renvoi à fErIO, mais ils utilisent un terme qui leur est commun (cheval), ce qui laisse entrevoir une réécriture à partir d'une source commune.

\section{Quatrième mode de la deuxième figure}

Le quatrième mode de ceux qui constituent la deuxième figure est celui qui, à partir d'une majeure affirmative universelle et d'une mineure particulière négative, a pour conclusion une particulière négative ${ }^{55}$.

Ce mode n'accepte aucune réduction directe, puisque l'universelle affirmative ne se convertit pas parfaitement et que la particulière négative ne se convertit pas du tout. L'universelle affirmative se convertit en particulière. Or, de deux prémisses particulières, on ne peut tirer aucune conclusion valide. De même d'ailleurs on ne peut rien tirer de deux r prémisses négatives. 
La démonstration de la validité de ce mode se fait au moyen d'un raisonnement par l'impossible. De ce raisonnement par l'impossible, je parlerai à la fin de cet ouvrage.

texte d'Athanase

Quelque être vivant n'est pas animal

Tout être sensible est animal

Quelque être vivant n'est pas sensible

soit

Tout être sensible est animal

Quelque être vivant n'est pas animal

Quelque être vivant n'est pas sensible

texte de Paul

Tout être instruit est homme

Quelque animal n'est pas homme

Quelque animal n'est pas instruit

texte de Probha

Tout homme est doué de raison

Quelque animal n'est pas doué de raison

Quelque animal n'est pas homme

texte de Pierre

Tout homme est animal

Quelque pierre n'est pas animal

Quelque pierre n'est pas homme

Les deux règles générales données ici par Athanase sont probablement des adjonctions témoignant d'écritures successives. Elles auraient mieux trouvé leur place dans la présentation générale. Si Athanase a ainsi complété son manuel au fil du temps, c'est parce qu'il l'a utilisé pour dispenser un enseignement, qui lui a permis de prendre conscience de lacunes au moment même où elles devenaient évidentes. Ce commentaire trouve une confirmation dans le rapport de la description du raisonnement par l'impossible à la fin du livre. Ainsi Athanase pourra-t-il expliquer ensemble la démonstration difficile de ce mode et celle du sixième mode de la troisième figure.

Pour ce mode, sans doute en raison des problèmes qu'il pose, les quatre logiciens ont innové dans le choix des termes. Ils semblent avoir eu pour ambition de rendre évident que la contradictoire de la conclusion serait fausse, voire absurde. 


\section{Troisième figure}

Dans la troisième figure, il y a six modes concluants.

Ce qui caractérise cette figure, c'est que la prémisse mineure est toujours affirmative et que le moyen terme est sujet dans les deux propositions.

\section{Premier mode de la troisième figure}

Le premier mode de la troisième figure est celui qui, à partir d'une affirmative universelle comme mineure et d'une affirmative universelle comme majeure, a pour conclusion une particulière affirmative. Par la conversion de la mineure, on retrouve la première figure ${ }^{56}$.

texte d'Athanase

Tout homme est animal

Tout homme est capable de rire

Quelque animal est capable de rire

soit

Tout homme est capable de rire

Tout homme est animal

Quelque animal est capable de rire

texte de Paul

Tout homme est doté de raison

Tout homme est animal

Quelque animal est doté de raison

texte de Probha

Tout homme est mortel

Tout homme est doté de raison

Quelque être doté de raison est mortel

texte de Pierre

Tout homme est substance

Tout homme est animal

Quelque animal est substance

Le choix fait par les quatre logiciens du même moyen terme (homme) est certainement l'indice d'une inspiration puisée dans un stock commun d'exemples usuels.

Probha renvoie directement au troisième mode de la première figure, tout comme Pierre. On trouve, d'autre part, chez Athanase un écho manifeste au syllogisme du troisième mode de la première figure chez Paul, ce qui constitue un indice supplémentaire de la circulation d'exemples consacrés par la pratique pédagogique ou savante. 


\section{Deuxième mode de la troisième figure}

Le deuxième mode de la troisième figure est celui qui, à partir d'une mineure affirmative universelle et d'une majeure négative universelle, a pour conclusion une particulière négative. On retrouve la première figure en convertissant la mineure ${ }^{57}$.

texte d'Athanase

Tout homme est animal

Nul homme n'est pierre

Quelque animal n'est pas pierre

soit

Nul homme n'est pierre

Tout homme est animal

Quelque animal n'est pas pierre

texte de Paul

Nul homme n'est quadrupède

Tout homme est mortel

Quelque mortel n'est pas quadrupède

texte de Probha

Nul homme n'est cheval

tout homme est mortel

Quelque mortel n'est pas cheval

texte de Pierre

Nul homme n'est pierre

Tout homme est animal

Quelque animal n'est pas pierre

Athanase et Pierre ont recours exactement au même exemple. On relève, d'autre part, chez Paul et Probha une indéniable ressemblance, puisque seul le grand terme est différent. De telles similitudes ne peuvent pas être fortuites.

Athanase s'intéresse, pour en finir avec ce mode, à la réduction au quatrième mode de la première figure.

Si l'on convertit la prémisse mineure Tout homme est animal en Quelque animal est homme, et si on associe cette proposition à la prémisse majeure Aucun homme n'est pierre, on conclut que Quelque animal n'est pas pierre.

\section{Troisième mode de la troisième figure}

Le troisième mode de la troisième figure est celui qui, à partir d'une affirmative universelle comme majeure et d'une particulière affirmative comme mineure conclut par une particulière affirmative. Par la conversion 
de la mineure, on retrouve la structure de la première figure ${ }^{58}$.

texte d'Athanase

Quelque animal est blanc

Tout animal est sensible

Quelque blanc est sensible

soit

Tout animal est sensible

Quelque animal est blanc

Quelque blanc est sensible

texte de Paul

Tout homme est animal

Quelque homme est noir

Quelque animal est noir

texte de Probha

Tout homme est mortel

Quelque homme est doté de raison

Quelque être doté de raison est mortel

texte de Pierre

Tout homme est substance

Quelque homme est animal

Quelque animal est substance

Les choix de Probha et de Pierre sont dictés par la volonté de rendre manifeste la nécessité de réduire ce mode au troisième mode de la première figure. On trouve des échos subtils entre les syllogismes d'Athanase et de Paul (animal moyen terme et grand terme ; noir et blanc petits termes).

Athanase explique la conversion. Quand on convertit la prémisse Quelque animal est blanc en la transformant en Quelque blanc est animal et que l'on associe cette prémisse à celle qui énonce que Tout animal est sensible, on conclut, d'après le troisième mode de la première figure, qui est parfait $^{59}$, Quelque blanc est sensible.

\section{Quatrième mode de la troisième figure}

Le quatrième mode de la troisième figure est celui qui, à partir d'une mineure affirmative universelle et d'une majeure particulière affirmative, aboutit à une particulière affirmative en guise de conclusion. Par conversion de la majeure et de la conclusion, on retrouve la structure de la première figure $^{60}$.

texte d'Athanase

Tout homme est animal

rV 
Quelque homme est instruit

Quelque animal est instruit

soit

Quelque homme est musicien

Tout homme est animal

Quelque animal est musicien

texte de Paul

Quelque bipède est homme

Tout bipède est animal

Quelque animal est homme

texte de Probha

Quelque homme est doté de raison

Tout homme est mortel

Quelque mortel est doté de raison

Texte de Pierre

Quelque homme est substance

Tout homme est animal

Quelque animal est substance

Les syllogismes de Probha et de Pierre ont les mêmes termes que ceux auxquels on les réduit dans la première figure. Ceux d'Athanase et de Paul ont des termes communs, pourvus d'une fonction différente (homme est moyen terme chez l'un, grand terme chez l'autre) ou identique (animal est petit terme dans les deux cas). Ces variations sont-elles liées à des reconstructions originales élaborées à partir d'un modèle commun ? Répétés, de tels échos doivent avoir une signification liée à l'histoire de l'enseignement oral ou écrit de l'analytique.

Athanase complète sa présentation par la description de la réduction à un mode de la première figure. Si l'on convertit la majeure Quelque homme est instruit en Quelque être instruit est homme et qu'on l'associe à l'autre prémisse, Tout homme est animal, on conclut que Quelque être instruit est animal d'après le troisième mode de la première figure.

\section{Cinquième mode de la troisième figure}

Le cinquième mode de la troisième figure est celui qui, à partir d'une mineure affirmative particulière et d'une majeure négative universelle, les termes étant associés conformément à la structure de la troisième figure, conclut par une négative particulière. Par conversion de la prémisse mineure affirmative particulière, on retrouve la structure de la première figure ${ }^{61}$.

texte d'Athanase

Quelque homme est blanc 


\section{Jean-Pierre Levet}

Nul homme n'est oiseau

Quelque <être> blanc n'est pas oiseau

soit

Nul homme n'est oiseau

Quelque homme est blanc

Quelque $<$ être $>$ blanc n'est pas oiseau

texte de Paul

Nul oiseau n'est cheval

Quelque oiseau est noir

Quelque <être $>$ noir n'est pas cheval

texte de Probha

Nul homme n'est cheval

Quelque homme est mortel

Quelque mortel n'est pas cheval

texte de Pierre

Nul homme n'est pierre

Quelque homme est animal

Quelque animal n'est pas pierre

Le syllogisme de Pierre a les mêmes termes que son exemple du quatrième mode de la première figure. Dans les trois syllogismes syriaques, on retrouve des termes communs (homme et cheval) et un jeu de couleurs (blanc, noir), qui sont autant d'échos intéressants.

De manière attendue, Athanase décrit la réduction à un mode de la figure parfaite.

Si l'on convertit la prémisse Quelque homme est blanc pour obtenir Quelque blanc est homme et que l'on associe cette proposition à l'autre prémisse, on conclut, d'après le quatrième mode parfait ${ }^{62}$ de la première figure, avec une négative particulière.

\section{Sixième mode de la troisième figure}

Le sixième mode de la troisième figure est celui qui est formé d'une mineure affirmative universelle et d'une majeure particulière négative. Sa conclusion est une particulière négative ${ }^{63}$.

On n'établit pas sa démonstration par une opération de conversion, mais au moyen d'un raisonnement par l'impossible, comme on le fait pour le deuxième mode de la deuxième figure.

En fait, la particulière négative ne se convertit pas et si l'on convertit une universelle affirmative, on obtient une particulière affirmative. A partir de deux particulières, on ne peut rien conclure dans aucune figure, tout rq 
comme on ne peut rien conclure à partir de deux négatives. Dans la deuxième figure, il faut que la qualité des propositions ne soit pas la même. Par la qualité, l'affirmative s'oppose à la négative et la négative à l'affirmative. Dans la troisième figure, la mineure est toujours affirmative.

Voilà brièvement ce qu'il y avait à dire des syllogismes des différentes figures.

\section{Appendice}

Ainsi la présentation des quatorze modes valides se termine-t-elle par une répétition, puisque les règles générales qui sont énoncées à propos du seizième mode l'ont déjà été lorsqu'il a été question du dernier mode de la deuxième figure. Un tel défaut s'expliquerait de manière satisfaisante par une adjonction liée à un enseignement oral conduisant à résumer des lois, éventuellement déjà indiquées, pour justifier la cohérence des faits expliqués.

Ce rappel représente une sorte de transition avec la suite immédiate du texte qui porte sur quelques considérations générales, que l'on a déjà traduites sous le titre d'Eléments additionnels. On a jugé bon de les décaler, précisément parce que le plan suivi par Athanase semble correspondre à une seconde version, complétée par des adjonctions correspondant à des lacunes relevées à l'occasion d'exposés pédagogiques oraux.

De toute manière, ces considérations adventices ont été insérées avant l'analyse du raisonnement par l'impossible, qui devrait, en bonne méthode, suivre la présentation du sixième mode de la troisième figure, annoncée à la fin du développement concernant le quatrième mode de la deuxième figure.

\section{Le raisonnement par l'impossible}

Le raisonnement par l'impossible se déploie quand, à partir de deux prémisses vraies, on aboutit à une conclusion vraie et que l'on souhaite démontrer la vérité de cette conclusion ${ }^{64}$.

On pose ${ }^{65}$ alors comme admise la contradictoire de cette conclusion, laquelle est fausse et impossible. Si l'on parvient à démontrer que ce que l'on a supposé est impossible et faux, sera vraie la contradictoire de cette proposition, celle qui représente la première conclusion, obtenue à partir de prémisses vraies.

Prenons le quatrième mode de la deuxième figure, celui dont on a dit qu'il était concluant et que cela était démontrable par l'impossible ${ }^{66}$. 


\section{Jean-Pierre Levet}

Le quatrième mode de la deuxième figure est celui qui, à partir d'une majeure affirmative universelle et d'une mineure particulière négative, a pour conclusion une particulière négative.

La démonstration ne passe pas par la conversion des prémisses, mais, comme on l'a dit, par une réduction à l'impossible.

Exemple $^{67}$ : Tout être sensible est animal, Quelque être vivant n'est pas animal. Ces deux prémisses sont vraies toutes les deux. D'elles, on tire une conclusion vraie, à savoir Quelque être vivant n'est pas sensible ${ }^{68}$.

Supposons que cette conclusion soit fausse. Dans un couple de contradictoires, si l'une est vraie, l'autre est fausse <et si l'une est fausse, l'autre est vraie $>$. <Dans notre supposition>, la contradictoire de cette conclusion serait donc vraie. Si l'on associe cette proposition $<$ Tout être vivant est sensible $>$ à l'autre prémisse $<$ la majeure $>$ qui énonce que Tout être sensible est animal, on conclut, en passant par le premier mode de la première figure, que Tout être vivant est animal, ce qui est faux <cela contredit la première mineure, dont on sait qu'elle est vraie $>$. Cela établit donc la vérité de la <première> conclusion particulière négative ${ }^{69}$.

En effet, le faux introduit dans la nouvelle conclusion ne peut pas procéder de la majeure, puisqu'il a été dit qu'elle était vraie ${ }^{70}$. Il faut donc le chercher dans la seconde mineure. Or, si elle est fausse, sera vraie sa contradictoire, qui énonce que Quelque être vivant n'est pas sensible, ce qui était précisément la conclusion du premier syllogisme.

Résumons. Si, par exemple, à partir de Tout être sensible est animal, Quelque être vivant n'est pas animal, on conclut que Quelque être vivant n'est pas sensible, la contradictoire de cette proposition ne peut pas être vraie. Voilà ce qu'est la démonstration par l'impossible.

Le sixième mode de la troisième ${ }^{71}$ figure appelle la même démonstration, à partir d'un raisonnement relatif à la conclusion. Si nous prenons la contradictoire de la conclusion, qui est une affirmative universelle, pour l'associer à la mineure du syllogisme, nous obtiendrons, en passant par le premier mode de la première figure, à partir de deux prémisses affirmatives universelles, une conclusion affirmative universelle. Or une telle conclusion est impossible ${ }^{72}$. Donc il faut bien conclure par une particulière. Si l'impossibilité s'est glissée dans la nouvelle prémisse posée, la contradictoire de cette dernière est vraie, or c'est la particulière négative $<$ c'est-à-dire la première conclusion> .

Quelque homme n'est pas grammairien, Tout homme est animal, donc Quelque animal n'est pas grammairien. Si cette conclusion était fausse, ए। 
serait vraie la proposition Tout animal est grammairien. Mais cela n'est pas possible $^{73}$.

\section{Synthèse et conclusion}

Tout ce qui a été présenté et commenté montre l'importance du choix des termes et des ressemblances de multiples formes qu'il permet de découvrir, ainsi que des influences, directes ou indirectes qu'il invite à soupçonner.

Pierre d'Espagne ne se sert que de quatre termes, homme, substance, animal, pierre, que l'on retrouve tous chez Probha et Athanase, trois d'entre eux figurant chez $\mathrm{Paul}^{74}$. Les logiciens d'expression syriaque recourent tous les trois à cheval. S'ils innovent parfois, ils se servent le plus souvent, en les disposant à leur manière dans des syllogismes concluants, de termes (blanc, noir, oiseau, animé, inanimé etc.) qui, comme cheval, prennent place dans les listes que propose Aristote pour l'illustration des modes invalides (avec les uns, il y a attribution, avec les autres, il n'y a pas attribution).

Aristote ne construit jamais les raisonnements qu'illustrent les termes dont il donne la liste. Ainsi fournit-il une sorte de vivier de termes, réutilisables au gré de ses interprètes. Les Syriaques y ont abondamment puisé. C'est là sans doute une des explications possibles des correspondances que l'on a relevées.

La rédaction même des Analytiques, œuvre acroamatique du corpus aristotélicien, rend le texte vivant, non figé, ni même définitif, puisque le lecteur est invité à construire les syllogismes dont les termes lui sont indiqués. Il est ainsi appelé à devenir vraiment, par l'étude, un chercheur de vérité.

Si l'on veut apporter une preuve supplémentaire à la démonstration que les versions des Syriaques sont plus des réécritures que des traductions proprement dites, on la trouvera dans l'exploitation qui a été faite des termes engagés dans la construction de prémisses destinées à aboutir à des conclusions valides, alors qu'Aristote les avait énumérés pour illustrer des modes non concluants.

Il s'est donc développé une vie propre de la syllogistique aristotélicienne dans l'ensemble du monde euro-méditerranéen. Chaque «traducteur» a pu ainsi se transformer en exégète discret et en commentateur implicite et subtil.

C'est bien cette vitalité de la syllogistique que l'on s'est efforcé de saisir principalement dans le monde d'expression syriaque. Impulsé 


\section{Jean-Pierre Levet}

consciemment $^{75}$ ou non ${ }^{76}$ par Aristote, ce dynamisme, d'essence pédagogique, se révèle remarquable : un contenu scientifique rigoureux se révèle compatible avec l'imagination, donc avec la liberté, des traducteurs potentiels.

Ainsi ces premiers chapitres des Analytica Priora, qui ont tant fécondé, et pendant de si nombreux siècles, les civilisations des deux bords de la Méditerranée, ont-ils permis à tous ceux qui les ont étudiés et rendus dans une autre langue que le grec d'user, en plus de talents de traducteurs, d'originalité et d'une faculté de mise en forme nouvelle ou de recréation. Mise en action nécessairement, en raison du silence d'Aristote sur ce point précis, par le choix des termes destinés à entrer dans les modes concluants, la liberté créatrice, encadrée puisqu'elle s'inspire très largement des listes données par Aristote dans une autre intention, celle de faire travailler les esprits pour bâtir des exemples invalides concrets, s'est tout naturellement étendue à la composition devenant recomposition, évidemment dans les limites permises par la matière traitée, dont le contenu est le plus rigoureux qui soit, puisque le vrai y entre dans une opposition binaire stricte avec le faux.

Il y a là de bonnes raisons pour soutenir que ces premiers chapitres des Analytica Priora méritent d'être considérés comme fournissant une contenu exemplaire en vue de l'élaboration d'une philologie euroméditerranéenne : grâce à eux, on peut saisir, pour ainsi dire de l'intérieur, la vie même d'un texte fondateur et l'activité des facultés intellectuelles de ceux qui l'ont traduit dans des langues appartenant à la même famille que le grec ou extérieures à cette famille.

Dans cette perspective, le syriaque et les maîtres qui l'ont utilisé doit occuper une place centrale, puisqu'il a servi d'intermédiaire entre le grec et le monde sémitique. Son rôle est identique à celui que le latin a joué en Occident dans la transmission de l'héritage grec.

Voilà pourquoi, si l'on souhaite vraiment qu'une philologie nouvelle soit construite autour de ce que la Grèce a légué aux civilisations qui se sont développées des deux côtés de la Méditerranée, faire revivre l'apport du syriaque est une entreprise urgente, ouvrant de nombreuses perspectives (traductions et commentaires, analyses linguistiques comparatives et contrastives impliquant les langues chamito-sémitiques et indoeuropéennes).

Grâce à Athanase de Balad, Paul le Perse, Probha et Pierre d'Espagne, c'est bien, autour d'Aristote, à des fondements de civilisations rT 
méditerranéennes que l'on s'est intéressé, en souhaitant donner l'impression de les avoir saisis dans leur dynamisme vital même.

Né près de Mossoul et parlant une langue, de grande et longue tradition, celle de l'araméen, aujourd'hui quasiment disparue, Athanase souhaitait contribuer au rayonnement de la civilisation grecque. Son exemple n'invite-t-il pas, à sa manière, les philologues contemporains à faire rayonner la civilisation euro-méditerranéenne dans le monde du $\mathrm{XXI}^{\mathrm{e}}$ siècle, qui a grand besoin de redécouvrir, au-delà des nombreuses disciplines qu'elle a mises en honneur, comme la médecine, qui suppose compassion et sympathie fraternelles envers tous les hommes, et comme la logique, dont le primat implique une passion constante pour l'étude et la vérité, les éléments majeurs de sagesse dont elle est porteuse ? 


\section{Jean-Pierre Levet}

\section{Les Notes :}

1 Elle sera publiée in extenso dans le tome 10 de la revue Tôzai en 2009.

2 Sur cette œuvre d'Athanase, on se reportera à Giuseppe Furlani, «Contributi alla storia della filosofia greca in Oriente, testi siriaci, VI, Una introduzione alla logica aristotelica di Atanasio di Baladh», Rendiconti della Reale Accademia dei Lincei, XXV, p. 717778 (édition du texte syriaque); «Sull'Introduzione di Atanasio di Baladh alla logica e syllogistica aristotelica», Atti del Reale Istituto Veneto di Scienza, Lettere ed Arti, LXXXI/2, 1921-1922, p. 635 à 644, et LXXXV/2, 1925-1926, p. 319-344; sur les traducteurs de la logique d'Aristote en syriaque, on consultera Ephrem-Isa Yousif, Les philosophes et traducteurs syriaques. D'Athènes à Bagdad, Paris, $1997=$ Yousif (1997) ; Javier Teixidor, Aristote en syriaque. Paul le Perse, logicien du VIe siècle, Paris, 2003 = Teixidor (2003); Henri Hugonnard-Roche, La logique d'Aristote du grec au syriaque, Paris, 2004 = Hugonnard-Roche (2004); Jean-Pierre Levet, «La syllogistique de Paul le Perse. Esquisse d'une étude de philologie euroméditerranéenne, Aristote, Paul et Probha », Tôzai, 9, 2007, p. 189-249 = Levet (2007).

3 Voir, par exemple, Dimitri Gutas, Greek Thought, Arabic Culture, Londres, 1998.

4 Sur Pierre d'Espagne, on se reportera à J.-P. Levet, «Pierre d'Espagne et la tradition scientifique grecque (logique et médecine) », Gengo Bunka, 9, mars 1992, p. 1 à $63=$ Levet (1992).

5 Voir Hugonnard-Roche (2004), p. 39.

6 Sur la fidélité au texte originel, voir Hugonnard-Roche (2004), p. 85.

7 Sur cette déclaration d'Athanase dans l'introduction de son traité, voir Teixidor (2003), p. 53.

8 Yousif (1997), p. 38 ; Levet (2007), p. 232-233.

9 Sur le traité de logique de William de Sherwood, voir Norman Kretzman, William of Sherwood's Introduction to Logic, Minneapolis, 1966 (1975).

10 Voir Levet (1992), p. 1-6, avec une bibliographie p. 60-63 ; Francis P. Dinnen, Peter of Spain, Language in Dispute, Amsterdam et Philadelphie, 1990.

11 L'édition de référence est celle de W. D. Ross, Aristotle's Prior and Posterior Analytics, Oxford, 1980.

12 Sur ces versions de Boèce, bien éditées par L. Minio-Paluello, Aristoteles Latinus, III, 14, Analytica Priora, Bruges et Paris, 1962, voir J.-P. Levet, « Philologie et Logique : Boèce traducteur des premiers chapitres du livre I des Analytica Priora d'Aristote», $R H T, 18,1988$, (1989), p. 1 à 62 ; les travaux que l'on a cités sur Boèce et Pierre d'Espagne correspondent à la publication de conférences données par J.-P. Levet au Collège de France sur invitation du professeur Jean Irigoin ; un programme d'étude développée de philologie méditerranéenne consisterait en un examen approfondi, mot après mot, du texte grec d'Aristote et de ses versions latines et syriaques.

13 Il se caractérise par une plus grande extension.

14 Il est d'extension plus petite.

15 Comprendre est marchant, est un être qui marche.

16 Comme Aristote, Athanase ne mentionne pas les singulières, qui sont assimilables à des universelles.

ro 
Athanase de Balad et la syllogistique catégorique d'Aristote en syriaque

17 Cette distinction ne renvoie pas nécessairement aux prémisses du syllogisme, car elle peut signifier que certaines propositions ont un prédicat universel (les majeures), à la différence des autres, les mineures.

18 L'exemple est d'Aristote (I, 2).

19 Aristote emploie un autre exemple.

20 L'exemple est imaginé par Athanase.

21 Athanase évoque les contraires (A et E), qui ne peuvent pas être vraies ensemble.

22 Il est ici question de la subcontrariété (I et O), qui ne peuvent pas être fausses ensemble.

23 Il s'agit d'une part de $\mathrm{A}$ et $\mathrm{O}$ et, d'autre part, de $\mathrm{E}$ et I.

$24 \mathrm{~A}, \mathrm{E}, \mathrm{I}, \mathrm{O}$.

25 Ce raisonnement analogique est fondé sur un exemple : Pisistrate est devenu tyran après avoir reçu une garde personnelle, donc une autre personne, en l'occurrence Denys de Syracuse, deviendrait également tyran si elle recevait une garde personnelle.

26 La démonstration syllogistique, à partir de prémisses vraies et selon des modes valides, est toujours vraie; les démonstrations de type inductif, qu'elles soient analogiques ou proprement inductives, le sont, elles, le plus souvent; on se persuade qu'elles sont vraies, sans en avoir la certitude absolue.

$27 \mathrm{Ce}$ raisonnement relève du premier mode de la première figure.

28 On passe par le deuxième mode valide de la première figure : Nul animal n'est pierre, or Tout homme est animal, donc Nul homme n'est pierre; on a démontré auparavant que Tout homme est animal par le premier mode de la première figure : Tout être sensible est animal, or Tout homme est <un> être sensible, donc Tout homme est animal.

29 Ces raisonnements correspondent aux deux syllogismes qu'Aristote qualifie de parfaits.

30 Athanase s'exprime de manière elliptique : en tant qu'intermédiaire, le connu est exprimé par le moyen terme, mais cela n'est pas précisé ; il n'est pas non plus indiqué que le majeur est le prédicat de la conclusion.

31 Athanase évoque ici la conversion parfaite de E et de I.

32 Athanase ne mentionne ni le contingent ni l'impossible.

33 Il est possible qu'un homme âgé de soixante ans soit blanc, soit ayant les cheveux blancs.

34 Athanase rappelle qu'il existe plusieurs types de propositions particulières négatives.

35 En retenant trois figures, Athanase, comme Paul et Probha, se place dans une perspective rigoureusement aristotélicienne.

36 Négligés par Aristote, les polysyllogismes se décomposent en syllogismes simples d'un nombre inférieur d'une unité à celui des prémisses posées.

37 Athanase n'évoque pas la structure de la conclusion (petit terme sujet, grand terme prédicat).

38 Elle contient les modes qu'Aristote qualifie de parfaits.

39 L'ordre des prémisses est mineure, puis majeure.

40 Substance, animal, homme.

41 Athanase évoque ainsi les syllogismes relevant des modes concluants (donc formellement véridiques) construits à partir d'une matière vraie (matériellement vrais).

42 Les propositions indéterminées sont quantitativement ambiguës.

43 Pour le détail, on se reportera aux traductions de Levet (2007).

44 Mode bArbArA. 
45 Voir la traduction de L'Institution Logique par J.-P. Levet, p. 267, in Galien, Traités philosophiques et logiques, Paris, 1998.

46 Mode cElArEnt.

47 Mode dArII.

48 Sensible, animal, être vivant.

49 Mode fErIO.

50 Mode cEsArE.

51 Réduction de cEsArE à cElArEnt par conversion parfaite de la majeure.

52 Mode cAmEstrEs.

53 Réduction de cAmEstrEs à cEsArE par conversion parfaite de la majeure et de la conclusion, complétée par une permutation des prémisses.

54 Mode fEstInO, réduit à fErIO par conversion parfaite de la majeure.

55 Mode bArOcO.

56 Mode dArAptI, réduit à dArII par conversion imparfaite de la mineure.

57 Mode fElAptOn, réduit à fErIO par conversion imparfaite de la mineure.

58 Mode dAtIsI, réduit à dArII par conversion parfaite de la mineure.

59 En fait seuls les deux premiers modes de cette première figure sont réellement parfaits, mais on peut facilement passer de AII à AAA en prenant en considération l'ensemble que forment les quelques éléments représentant le petit terme.

60 Mode dIsAmIs, réduit à dArII par conversion parfaite de la majeure et de la conclusion, avec permutation des prémisses.

61 Mode fErIsOn, que l'on réduit à fErIO par conversion parfaite de la mineure affirmative particulière.

62 Ce quatrième mode n'est pas parfait, mais on le réduit facilement au deuxième mode, qui, lui, est réellement parfait; on procède, mutatis mutandis, comme on l'a fait pour passer de dArII à bArbArA.

63 Mode bOcArdO.

64 En d'autres termes, la matière étant vraie, on veut vérifier la validité formelle de l'inférence.

65 Il s'agit d'une supposition dont la fausseté sera démontrée.

$66 \mathrm{Il}$ s'agit de bArOcO.

67 Athanase reprend l'exemple qu'il a proposé.

68 On souhaite donc bien vérifier si c'est l'inférence qui est véridique.

69 On pose Tout être sensible est animal, majeure vraie du premier syllogisme; Tout être vivant est sensible, nouvelle mineure, contradictoire de la première conclusion; on obtient ainsi un syllogisme du premier mode de la première figure, dont la conclusion est Tout être vivant est animal, ce qui est la contradictoire de la première mineure, vraie quant à elle ; cette conclusion nouvelle est donc fausse ; le faux ayant été introduit par la contradictoire de la première conclusion, il est démontré ainsi que celle-ci était vraie et donc que l'inférence est formellement valide.

70 Le faux ne peut pas non plus avoir été introduit par le mode, valide et même parfait, du second syllogisme.

71 Mode bOcArdO.

72 Elle est la contradictoire de la majeure initiale. 
= Athanase de Balad et la syllogistique catégorique d'Aristote en syriaque

73 Tout animal est grammairien, contradictoire de la première conclusion, est utilisée pour construire un nouveau syllogisme du premier mode de la première figure avec la première mineure, Tout animal est grammairien, Tout homme est animal ; on conclut que Tout homme est grammairien, ce qui est la contradictoire de la première majeure, vraie; la conclusion du second syllogisme est porteuse d'une fausseté héritée de la nouvelle majeure ; puisque celle-ci est fausse, est vraie sa contradictoire, c'est-à-dire la conclusion du premier syllogisme, dont on souhaitait établir la vérité pour garantir la validité de l'inférence.

74 Mais dans le texte de ce dernier figurent les modes invalides, que l'on n'a pas examinés, parce qu'ils sont absents des œuvres d'Athanase et de Probha.

75 Pour le monde grec.

76 Aristote ne pouvait évidemment pas prévoir que son texte serait traduit en syriaque et en latin. 